\title{
A preliminary study of personality traits and their influence on software piracy
}

\begin{abstract}
Software piracy worldwide has resulted in serious industrial, academic and economic consequences. However, the problem can be solved if the root causes can be identified. Personality traits are said to be an effective positive influence on unethical behaviour. For this reason, personality traits could be a predictor for software piracy. This study investigates personality traits as a factor in students intention to pirate software by enhancing the Theory of Planned Behaviour (TPB) model. The objective of this current research is to provide a preliminary test on the personality traits with the TPB model in software piracy behaviour in order to determine the reliability and validating instruments. The personality traits used in this study were extraversion, agreeableness, conscientiousness, neuroticism and openness according to Big Five Inventory (BFI). A survey was conducted to achieve the research objective. Questionnaire was carried out on 40 computer sciences students to determine how reliable and valid it would be to proceed with further research. This study has been compared with previous studies, and several minor modifications of the instrument were made. All the instruments were validated and applied in the actual study. The results of factor analyses confirm and refine the reliability of the instruments for both big five personality variables and TPB elements in software piracy. The findings could also be helpful to information systems practitioners and academics in the implementation of measures to reduce the incidence of software piracy among students.
\end{abstract}

Keyword: TPB model; Personality traits; Software piracy 